\title{
The limits to exercise performance and the future of fatigue research $^{\star}$
}

\author{
F E Marino, M Gard, E J Drinkwater \\ School of Human Movement Studies, Charles Sturt University, Bathurst, New South Wales, Australia \\ Correspondence to: Professor Frank E Marino (fmarino@csu.edu.au)
}

\begin{abstract}
The study of human fatigue stretches back centuries and remains a significant part of medical and social discourse. In the exercise sciences fatigue is routinely related to the ability to produce muscle force or to the recovery from force decrements. However, the study of fatigue has by virtue of the experimental paradigm excluded the subjective sense a person attributes to an event or experience, thus reducing our overall understanding of the fatigue process. Modern studies report the causes of fatigue as either central or peripheral in origin. Although useful, this dichotomy can also exclude the individual subjective assessment. Furthermore, adhering dogmatically to set parameters is likely limiting the advancement of our understanding. A more realistic paradigm would permit the individual to use the sensory cues to adjust the effort along with the fatigue process rather than rely purely on feedback mechanisms. Therefore, bringing feedforward mechanisms of the brain into fatigue research perhaps represents the next phase in the unravelling of the fatigue process.
\end{abstract}

\section{THE HISTORY AND MEANING OF FATIGUE}

It is immensely difficult to provide a detailed historical account of the development of fatigue as a concept in the exercise sciences. However, the study of fatigue stretches back centuries to scientists such as Galvani, who provided the ideas and tools to undertake experiments related to the electrical impulses needed to animate skeletal muscles. ${ }^{1}$ The definitive work of Mosso in the 18th Century stands as a landmark in the study of fatigue. ${ }^{2}$ In his book La fatica (fatigue), ${ }^{2}$ he concluded that there were two phenomena which categorised fatigue: "The first is the diminution of the muscular force. The second is fatigue as a sensation. That is to say, we have a physical fact which can be measured and compared, and a psychic fact which eludes measurement" ( $\mathrm{p}$ 154). Notably, early textbooks such as Physiology of muscular exercise by Bainbridge in $1931^{3}$ pointed out that the limit of exercise "has often been ascribed to the capacity of the heart alone, but the facts as a whole indicate that the sum of the changes taking place throughout the body brings about the final cessation of effort" ( $\mathrm{p} 176)$. It is an interesting fact that research into fatigue is highly complex, and consensus about the aetiology of this human condition still evades us. Not surprisingly, even after centuries of research in this area, fatigue is

${ }^{*}$ This article was reproduced with permission from the BMJ Group. It was originally published in the British Journal of Sports Medicine 2011;45:65-67 (originally published online 2 December 2009). still very much a part of medical and social discourse. There may be several reasons for this, not least of which could be the loss or change in the meaning of the term fatigue. The Oxford Dictionary ${ }^{4}$ defines fatigue as "extreme tiredness after exertion; reduction in efficiency of a muscle, organ etc. after prolonged activity". Compare this definition to that of exhaustion which is often used interchangeably by exercise physiologists and which is defined as "a total loss of strength; to consume or use up the whole of". ${ }^{4}$ Clearly, these are substantially different meanings. In addition to these general meanings, there is wide variation in definitions of fatigue in the exercise sciences which include statements such as "the failure to maintain the required or expected force," ${ }^{5}$ or "a loss of maximal force generating capacity"6 or "a reversible state of force depression, including a lower rate of rise of force and a slower relaxation"? There are many more statements or definitions like these which attempt to capture the specific observation that there has been a decline in the ability to produce skeletal muscle tension of a given magnitude in order to quantify the amount of fatigue that has developed.

Recently, it has been argued that meaning itself, the subjective sense a person attributes to an event or experience, tends to be overlooked in scientific research. As a result, we are left with a naïve understanding about the underlying mechanisms so that the subjective experience is nothing more than a series of brain states. ${ }^{8}$ The study of fatigue is an example of how scientific rules which ordinarily have finite meanings have constrained the pursuit of understanding the fatigue experience.

\section{CENTRAL OR PERIPHERAL FATIGUE?}

Much of what is reported in the literature about the mechanisms of fatigue is related to the reductionist and sometimes mutually exclusive dichotomy of either peripheral or central mechanisms with conclusions usually drawn about the contribution of each to the total outcome. One might argue that such a dichotomous paradigm is appropriate since it provides insights about the processes at specific sites. From these site-specific changes, one can apparently conclude whether the site of fatigue is located in the central nervous system or peripherally at or below the neuromuscular junction. However, over a century ago, Mosso wrote that "It is not will, not the nerves, but it is the muscle that finds itself worn out after the intense work of the brain". ${ }^{2}$ Mosso expressly noted that the "fatigue of brain reduces the strength of the muscles". This is perhaps one of the initial statements in this area of work which gave rise to the central versus peripheral paradigm and it may be this "either/or" approach that limits our investigations and understanding of fatigue. Moreover, this dichotomy excludes the obvious individual subjective assessment of fatigue. For example, during high intensity exercise of short duration, the fatigue that develops is thought to be primarily peripheral in origin so that the ability of the muscle to generate further tension is reduced because of changes to the properties of the surface membrane 
of the muscle fibre, the process of calcium release or the function of excitation-contraction coupling ${ }^{9}$ (pp 105-119). However, if the familiar feelings or sensations of "burning" that develop during this process have no meaning for the individual and play no role, then this removes completely the possibility that the individual is not adjusting their effort either consciously or subconsciously during the exercise. It seems intuitive that individuals would have some say over the way in which they deal with the fatigue that develops during this short intense exercise bout. However, the evidence for this is lacking probably because the protocols used to study fatigue do not allow for the individual to adjust their response relative to the feedback which might be available during exercise. It is because most of these tests are externally driven by an experimenter dictating the pace by, for example, a motorised treadmill that these tests are referred to as "brainless". 10

\section{DEFINITIONS AND MODELS OF FATIGUE}

There is an attraction to reaching a succinct and widely accepted definition of fatigue. Such a definition would be convenient for scientific investigation by providing a metric upon which to compare results. To base much of the sports and exercise science research on firmer footing could also add credibility to the discipline among the other natural sciences that have much more standardised metrics and terms of reference. However, to prematurely arrive at a definition that is only accepted because no better one exists may only confirm our own biases and misrepresent the reality of fatigue. For example, concise and intuitive theory has led us to routinely, but erroneously, explain fatigue with "lactic acid" for many decades. ${ }^{11}$ As a consequence, decades of research estimating metabolic acidosis and buffering on "lactic acid" is based on flawed theory. Considering our innate desire to understand a phenomenon, explaining fatigue with "lactic acidosis" persists ${ }^{12}$ even though there is now a gaping hole in our understanding of metabolic acidosis.

Similarly, that fatigue is based on feedback mechanisms has existed since Hill's so-called "Catastrophe Model" of fatigue. ${ }^{13}$ The interpretations of $\mathrm{VO}_{2}$ max testing made by Hill have guided how we quantify, understand and explain fatigue. That a model is concise or intuitive does not mean that we should cease looking to develop a better one, particularly when weaknesses in the Catastrophe Model come to light. ${ }^{14}$ While the "Central Governor Theory" ${ }^{15}$ might not be the final explanation of fatigue, ${ }^{16}$ a feedforward mechanism presents what is potentially the next step in advancing our understanding of fatigue. Considering that Hill's classical theory has undergone very little modification since its inception in the 1920s, is it possible that the constraints of this theory are contributing to limiting our understanding of fatigue?

A feedforward mechanism for explaining fatigue attempts to encompass events in which fatigue exists without exhaustion. The term "exhaustion" here does not necessary imply complete physical collapse but simply the inability to maintain the prescribed task (ie, task failure). ${ }^{17}$ Our current understanding of fatigue based on Hill's model cannot help us understand fatigue that involves voluntarily paced events like cycling time trials ${ }^{18}$ or team sports ${ }^{19}$ nor the fatigue associated with disease states. ${ }^{20}$ While most sporting events terminate with the occurrence of an event that is externally controlled (eg, the end of the race, expiry of the game clock), laboratory research investigating fatigue usually begins with no known end target and ends with voluntary termination. Athletes must consciously self-pace themselves to time the end of the event with fatigue while maintaining optimal performance. ${ }^{21} 22$ By removing conscious control over pacing, tests like the $\mathrm{VO}_{2}$ max are not specific to the phenomenon of self-paced events. ${ }^{13}$ Applying research results from externally paced tests to help us understand fatigue during self-paced events is entirely inappropriate.

It is possible that a multitude of explanations for fatigue exist, ranging from metabolic disturbances in the motor unit to mechanisms that are centrally mediated. ${ }^{23}$ The balance of central and peripheral mechanisms may be partially dependent upon duration/intensity of fatigue and the muscle group being assessed. ${ }^{24}$ However, that they are all based on feedback mechanisms seems to be a limiting factor in how we explain fatigue, particularly when considering the feedforward mechanisms necessary for the anticipation of fatigue and pacing strategies for self-paced events. While the nature of modern research often requires progressively greater specialization and refinement of theories, opportunities must be taken to discuss fatigue from a holistic perspective so that we can integrate different components of fatigue into a modern understanding.

\section{AT LEAST TWO FUTURE DIRECTIONS}

The future of research into fatigue during exercise points towards two potential lines of inquiry. First, there is the question of what we mean by the term fatigue. It is now clear that it can mean different things to different people. Most obvious here is the point that an organism might be said to be "fatigued" according to a wide range of criteria. However, perhaps, a more fundamental point is that fatigue can refer to both states and processes and that these need to be considered both separately and synergistically. Clearly, there will be times when an atomistic or reductive approach to understanding fatigue states and processes will be advantageous and other times when new knowledge will depend on abandoning the comfort of disciplinary, methodological or philosophical specialisation.

A second potential future for fatigue research concerns the kinds of questions we are prepared and able to ask. For example, researchers interested in what we might broadly call the limits to performance are familiar with two widely accepted but incompatible propositions. First, there is evidence that without "artificial" aids such as drugs or genetic manipulation we are unlikely to see significant future improvement in world records for sports such as swimming and track and field. ${ }^{25}$ On the other hand, there are those who point to feats of super-endurance, such as those by the first arctic explorers or the early trans-continental race walkers, as evidence that we cannot really know how far or fast we might go because we are rarely faced with circumstances that threaten our very survival. ${ }^{26}$ Taken together, these two propositions beg the question: are the limits to performance mechanical and immutable or fuzzy and suggestible?

One way of managing the tension that is created by these points of view is simply to avoid it. For example, we might argue that if modern athletes were motivated by the fear of starvation and/or death, they would run faster. Of course, this is sheer speculation at best. Hypothetically, there is also the problem that this kind of "encouragement" might have the opposite effect. Alternatively, we might argue that events such as the marathon and, say, walking 1000 $\mathrm{km}$ are both quantitatively and qualitatively different. In other words, it is not simply that the $1000-\mathrm{km}$ race is longer than the marathon; to put it crudely, it could be that the limits to performance in a $1000-\mathrm{km}$ race are predominantly mental, while the limits to performance in the marathon are predominantly physiological. This explanation has appeal, but there is always the lurking problem of differentiating the "mental" from the "physiological". 
A third solution might be to say that it depends on the "maturity" of the event. Ergo, in a highly competitive event such as the marathon, where lots of people are doing it and the prizes for winning are high, we reach the "limits" to performance fairly quickly (say, 100 years or so). However, super-endurance races are less popular, and, for this reason, we are proportionately much further away from the "limits" of performance in them. It is little wonder, then, that the limits to performance in this context appear to be "mental". For example, consider the rate of collapse of ultramarathon runners after the finish of the race or nearer the times to win medals. ${ }^{27}$

None of these resolutions to the limits of performance dilemma seem adequate. Perhaps, the problem here is that we now find ourselves pushing at a different set of limits the limits of the concepts we use and, in turn, the limits they place on the questions we are capable of asking. Perhaps, some of the dilemmas we currently face concerning the nature of fatigue are an artefact of our reliance on concepts like "physiological", "mental" and "psychological". Are these terms adequate anymore?

What is clear is that we can no longer pretend that conscious and unconscious decision making plays no role in fatigue states and processes. This means that studying fatigue as a closed feedback loop will no longer suffice. At this stage in our understanding of the brain, bringing feedforward mechanisms of the brain into fatigue research has the obvious potential to make our work much more "fuzzy" and much less "mechanical". For some, a fuzzy future will look too different from the past and make them turn away. For others, the possibility of inventing a new conceptual landscape with which to investigate/ unravel fatigue beckons.

Patient consent Not needed.

Contributors Each section of the manuscript was written by each of the authors.

Provenance and peer review Not commissioned; externally peer reviewed.

\section{REFERENCES}

1. Dougan A. Raising the dead: the men who created Frankenstein. Edinburgh: Birlinn Limited, 2008
2. Mosso A. Fatigue. London: Swan Sonnenschein \& Co Ltd, 1904

3. Bainbridge FA. The physiology of muscular exercise. 3rd edn. New York, USA: Longmans, Green and Co, 1931.

4. Moore B, editor. The Australian concise Oxford dictionary. 4th edn. South Melbourne: Oxford University Press, 2004.

5. Edwards RHT. Human muscle function and fatigue. In: Porter R, Whelan J, eds. Human muscle fatigue: physiological mechanisms. London: Pitman Medical, 1981:1-18.

6. Bigland-Ritchie B, Furbush F, Woods JJ. Fatigue of intermittent submaximal voluntary contractions: central and peripheral factors. J Appl Physiol 1986;61:421-9.

7. Fitts RH, Holloszy JO. Effects of fatigue and recovery on contractile properties of frog muscle. J Appl Physiol 1978;45:899-902.

8. Kretchmar RS. What to do with meaning? A research conundrum for the $21^{\text {st }}$ century. Quest 2007;59:373-83.

9. Jones D, Round J, de Haan A. Skeletal muscle: from molecules to movement. London: Churchill-Livingston, 2004

10. Noakes TD. Testing for maximum oxygen consumption has produced a brainless model of human exercise performance. Br J Sports Med 2008;42:551-5.

11. Robergs RA, Ghiasvand F, Parker D. Biochemistry of exercise-induced metabolic acidosis. Am J Physiol Regul Integr Comp Physiol 2004;287:R502-16.

12. Nakamura FY, Soares-Caldeira LF, Laursen PB, et al. Cardiac autonomic responses to repeated shuttle sprints. Int J Sports Med 2009;30:808-13.

13. Crewe $H$, Tucker R, Noakes TD. The rate of increase in rating of perceived exertion predicts the duration of exercise to fatigue at a fixed power output in different environmental conditions. Eur J Appl Physiol 2008;103:569-77.

14. Ansley L, Robson PJ, St Clair Gibson A, et al. Anticipatory pacing strategies during supramaximal exercise lasting longer than 30 s. Med Sci Sports Exerc 2004;36:309-14

15. Bam J, Noakes TD, Juritz J, et al. Could women outrun men in ultramarathon races? Med Sci Sports Exerc 1997;29:244-7.

16. Weir JP, Beck TW, Cramer JT, et al. Is fatigue all in your head? A critical review of the central governor model. Br J Sports Med 2006;40:573-86; discussion 586.

17. Hunter SK, Duchateau J, Enoka RM. Muscle fatigue and the mechanisms of task failure. Exerc Sport Sci Rev 2004;32:44-9.

18. Marino FE. Heat reactions in multiple sclerosis: an overlooked paradigm in the study of comparative fatigue. Int J Hyperthermia 2009;25:34-40.

19. Duffield R, Coutts AJ, Quinn J. Core temperature responses and match running performance during intermittent-sprint exercise competition in warm conditions. J Strength Cond Res 2009;23:1238-44.

20. Mock V, Atkinson A, Barsevick A, et al.; National Comprehensive Cancer Network NCCN Practice Guidelines for Cancer-Related Fatigue. Oncology 2000;14:151-61.

21. Gabbett TJ. Influence of fatigue on tackling technique in rugby league players. J Strength Cond Res 2008;22:625-32.

22. Royal KA, Farrow D, Mujika I, et al. The effects of fatigue on decision making and shooting skill performance in water polo players. J Sports Sci 2006;24:807-15.

23. Enoka RM, Duchateau J. Muscle fatigue: what, why and how it influences muscle function. J Physiol (Lond) 2008;586:11-23.

24. Behm DG, St-Pierre DM. Effects of fatigue duration and muscle type on voluntary and evoked contractile properties. J Appl Physiol 1997;82:1654-61.

25. Nevill AM, Whyte G. Are there limits to running world records? Med Sci Sports Exerc 2005;37:1785-8.

26. Noakes TD. The limits of endurance exercise. Basic Res Cardiol 2006;101:408-17.

27. Holtzhausen LM, Noakes TD, Kroning B, et al. Clinical and biochemical characteristics of collapsed ultra-marathon runners. Med Sci Sports Exerc 1994;26:1095-101. 